\title{
Pointwise multipliers of Musielak-Orlicz spaces and factorization
}

\author{
Karol Leśnik ${ }^{1} \cdot$ Jakub Tomaszewski ${ }^{1}$
}

Received: 26 November 2018 / Accepted: 25 May 2020 / Published online: 18 June 2020

(c) The Author(s) 2020

\begin{abstract}
We prove that the space of pointwise multipliers between two distinct Musielak-Orlicz spaces is another Musielak-Orlicz space and the function defining it is given by an appropriately generalized Legendre transform. In particular, we obtain characterization of pointwise multipliers between Nakano spaces. We also discuss factorization problem for Musielak-Orlicz spaces and exhibit some differences between Orlicz and Musielak-Orlicz cases.
\end{abstract}

Keywords Nakano spaces · Musielak-Orlicz spaces · Pointwise multipliers · Factorization

Mathematics Subject Classification 46E30 - 46B42

\section{Introduction}

Given two function spaces $X$ and $Y$ (over the same measure space), the space of pointwise multipliers $M(X, Y)$ is the space of all functions $f$ such that $f g \in Y$ for each $g \in X . M(X, Y)$ may be regarded as a generalized Köthe dual space (cf. [3,17]) and a basic question is to identify $M(X, Y)$ for a given spaces $X$ and $Y$. Many authors have investigated this problem for Orlicz spaces and many characterizations (mainly partial) have been given-see for example Shragin [23], Ando [1], O’Neil [22], ZabreikoRutickii [24], Maurey [18], Maligranda-Persson [17] and Maligranda-Nakai [16]. In 2000 Djakov and Ramanujan settled the problem for Orlicz sequence spaces and, recently, in [13] the authors established an analogous characterization for Orlicz func-

Jakub Tomaszewski
jakub.tomaszewski@ put.poznan.pl
Karol Leśnik
klesnik@vp.pl
$1 \quad$ Institute of Mathematics, Poznań University of Technology, ul. Piotrowo 3a, 60-965 Poznan, Poland 
tion spaces. In both cases, the space of pointwise multipliers $M\left(L^{\varphi_{1}}, L^{\varphi}\right)$ between Orlicz spaces is proved to be just another Orlicz space, i.e.

$$
M\left(L^{\varphi_{1}}, L^{\varphi}\right)=L^{\varphi \ominus \varphi_{1}},
$$

where the function $\varphi \ominus \varphi_{1}$ is generalized Young conjugate (generalized Legendre transform) of $\varphi_{1}$ with respect to $\varphi$. Observe that the above characterization generalizes, in the evident way, the classical Köthe duality formula for Orlicz spaces, this is

$$
\left(L^{\varphi_{1}}\right)^{\prime}=M\left(L^{\varphi_{1}}, L^{1}\right)=L^{\varphi_{1}^{*}}
$$

where $\varphi_{1}^{*}$ is the Young conjugate of $\varphi_{1}$ (i.e. $\varphi_{1}^{*}=i d \ominus \varphi_{1}$ ). Let us also mention here, that the identification as in (1.1) seems to be the most desirable, since the function $\varphi \ominus \varphi_{1}$ is given in an explicit and constructive way, in contrast to theorems from [16] and [11], which have rather existential character (cf. [1,18,22-24]).

In the paper we focus on the multipliers of Musielak-Orlicz spaces. Such investigations have been already initiated by Nakai [20] (cf. [21]). Under a number of assumptions on functions $\varphi, \varphi_{1}$ he generalized results of [16] to the Musielak-Orlicz setting. Since this method is not constructive (see discussion in [13]), we are not going to employ it. Instead of that we will use ideas of [5] and [13] to prove that the representation (1.1) holds also in the Musielak-Orlicz case, for an arbitrary $\sigma$-finite measure space and without any additional assumptions on Musielak-Orlicz functions $\varphi, \varphi_{1}$.

The paper is organized as follows. In Sect. 2 we give necessary definitions on Banach ideal space and Musielak-Orlicz spaces. We also define the function $\varphi \ominus \varphi_{1}$ (Young conjugate of $\varphi_{1}$ with respect to $\varphi$ ) for Musieak-Orlicz functions $\varphi, \varphi_{1}$.

The next section contains a number of technical lemmas concerning MusielakOrlicz spaces and multipliers. Consequently, we are ready to prove the representation theorem in the third section. Finally, the last section is devoted to discussion on factorization and differences between Orlicz and Musielak-Orlicz cases. In particular, we give an example showing that inequality $\varphi_{1}^{-1}\left(\varphi \ominus \varphi_{1}\right)^{-1} \succ \varphi^{-1}$ is not necessary condition for factorization of Musielak-Orlicz spaces, unlike in the Orlicz spaces case (cf. [13, Theorem 2]).

\section{Notation and preliminaries}

Trough the paper we will assume that $(\Omega, \Sigma, \mu)$ is a $\sigma$-finite, complete measure space. For a given set $A \in \Sigma$ we will denote the non-atomic part and purely atomic part of $A$ by $A^{c}$ and $A^{a}$, respectively. Notice, that there may be at most countably many atoms in $\Omega$, since the measure space is $\sigma$-finite. To simplify the notion, we will assume that all atoms in $\Omega$, if there are any, are singletons.

Let $L^{0}=L^{0}(\Omega, \Sigma, \mu)$ be the space of classes of equivalence of $\mu$-measurable, real valuable and $\mu$-a.e. finite functions. A Banach space $X \subset L^{0}$ is called the Banach ideal space if it satisfies the ideal property, i.e. $x \in L^{0}, y \in X$ and $|x| \leq|y|$ implies $x \in X$ and $\|x\|_{X} \leq\|y\|_{X}(|x| \leq|y|$ means that $|x(t)| \leq|y(t)|$ for $\mu$-a.e. $t \in \Omega)$. 
For $x \in L^{0}$ we define its support as $\operatorname{supp}(x):=\{t \in \Omega: x(t) \neq 0\}$. A support $\operatorname{supp} X$ of a Banach ideal space $X$ is defined as a measurable subset of $\Omega$ such that: (i) for each $x \in X$ there is $A \in \Sigma$ with $\mu(A)=0$ such that $\operatorname{supp}(x) \subset \operatorname{supp} X \cup A$, (ii) there is $x \in X$ such that $\mu(\operatorname{supp} X-\operatorname{supp}(x))=0$.

Notice that according to the above definition $\operatorname{supp} X$ is not unique, thus we rather write a support, than the support of $X$.

For any measurable $F \subset \Omega$ and a Banach ideal space $X$ we define

$$
X[F]:=\{x \in X: \mu(\operatorname{supp}(x) \backslash F)=0\} \text { with the norm }\|x\|_{X[F]}=\left\|x \chi_{F}\right\|_{X} .
$$

Given a Banach ideal space $X$ on $\Omega$ and a positive measurable weight function $v$, the weighted space $X(v)$ is defined as

$$
X(v):=\left\{x \in L^{0}: x v \in X\right\} \text { with the norm }\|x\|_{X(v)}=\|x v\|_{X} .
$$

Writing $X=Y$ for two Banach ideal spaces $X, Y$ we mean that they are equal as set, but norms are just equivalent. Recall also that for Banach ideal spaces $X, Y$ the inclusion $X \subset Y$ is always continuous, i.e. there is $c>0$ such that $\|x\|_{Y} \leq c\|x\|_{X}$ for each $x \in X$.

A Banach ideal space $X$ satisfies the Fatou property $(X \in(F P)$ for short) if for each sequence $\left(x_{n}\right) \subset X$ satisfying $x_{n} \uparrow x \mu$-a.e. and $\sup _{n}\left\|x_{n}\right\|_{X}<\infty$, there holds $x \in X$ and $\|x\|_{X} \leq \sup _{n}\left\|x_{n}\right\|_{X}$.

Given two Banach ideal spaces $X, Y$ over the same measure space $(\Omega, \Sigma, \mu)$ we define their pointwise product space

$$
X \odot Y=\left\{x \cdot y \in L^{0}: x \in X, y \in Y\right\},
$$

with a quasi-norm

$$
\|z\|_{X \odot Y}=\inf \left\{\|x\|_{X}\|y\|_{Y}: z=x y\right\} .
$$

If additionally $\operatorname{supp} X=\Omega$, then the space of pointwise multipliers from $X$ to $Y$ is defined as

$$
M(X, Y)=\left\{y \in L^{0}: x y \in Y \text { for all } x \in X\right\},
$$

with the natural operator norm

$$
\|y\|_{M}=\sup _{\|x\|_{X} \leq 1}\|x y\|_{Y}
$$

When there is no risk of confusion we will just write $\|\cdot\|_{M}$ for the norm of $M(X, Y)$.

If Banach ideal spaces $X$ and $Y$ have the Fatou property then both spaces $M(X, Y)$ and $X \odot Y$ have the Fatou property $[11,12,17]$.

We will need the following easy observation concerning the space of pointwise multipliers. Let $A, B \subset \Omega$ be measurable sets such that $A \cup B=\Omega$. Given a Banach ideal space $X$ over $\Omega$, we can decompose it as

$$
X=X[A] \oplus X[B],
$$


with the (equivalent) norm given by $\|x\|_{X[A] \oplus X[B]}=\left\|x \chi_{A}\right\|_{X[A]}+\left\|x \chi_{B}\right\|_{X[B]}$. It is easy to see that the space of pointwise multipliers respects such a "decomposition", i.e. $M(X, Y)$ may be written as follows

$M(X, Y)=M(X[A] \oplus X[B], Y[A] \oplus Y[B])=M(X[A], Y[A]) \oplus M(X[B], Y[B])$

In another words, determining the space of pointwise multipliers between two Banach ideal spaces, we may determine it on $A$ and on $B$ separately.

A function $\varphi:[0, \infty) \rightarrow[0, \infty]$ will be called the Young function if it satisfies $\varphi(0)=0, \lim _{u \rightarrow \infty} \varphi(u)=\infty$ and is convex on $\left[0, b_{\varphi}\right)\left(\right.$ or on $\left[0, b_{\varphi}\right]$ when $\left.\varphi\left(b_{\varphi}\right)<\infty\right)$, where

$$
b_{\varphi}=\sup \{u \geq 0: \varphi(u)<\infty\} .
$$

We point out here that we allow $\varphi(u)=\infty$ for each $u>0$. In such a case the corresponding Orlicz space $L^{\varphi}$ contains only the zero function.

Given a measure space $(\Omega, \Sigma, \mu)$, a function $\varphi: \Omega \times[0, \infty) \rightarrow[0, \infty]$ is called the Musielak-Orlicz function if the following conditions hold:

1. $\varphi(t, \cdot)$ is a Young function for $\mu$-a.e. $t \in \Omega$,

2. $\varphi(\cdot, u)$ is a measurable function for each $u \in[0, \infty)$.

Let $\varphi$ be a Musielak-Orlicz function. We define the convex modular $I_{\varphi}$ on $L^{0}$ as

$$
I_{\varphi}(x)=\int_{\Omega} \varphi(t,|x(t)|) d \mu(t) .
$$

The Musielak-Orlicz space $L^{\varphi}$ is defined as

$$
L^{\varphi}=\left\{x \in L^{0}: I_{\varphi}(\lambda x)<\infty \text { for some } \lambda>0\right\}
$$

and is equipped with the Luxemburg-Nakano norm

$$
\|x\|_{\varphi}=\inf \left\{\lambda>0: I_{\varphi}\left(\frac{x}{\lambda}\right) \leq 1\right\} .
$$

It is known that Musielak-Orlicz spaces have the Fatou property. Moreover, it follows immediately from the definition, that $\operatorname{supp} L^{\varphi}=\left\{t \in \Omega: b_{\varphi}(t)>0\right\}$ (up to a set of measure zero). In case, $\varphi$ does not depend on $t$, the Musielak-Orlicz space $L^{\varphi}$ is just the Orlicz space.

For a given Musielak-Orlicz function $\varphi$ we define two useful (functions) parameters

$$
\begin{aligned}
& a_{\varphi}(t)=\sup \{u \geq 0: \varphi(t, u)=0\} \\
& b_{\varphi}(t)=\inf \{u \geq 0: \varphi(t, u)=\infty\}
\end{aligned}
$$

It is known, that both $a_{\varphi}$ and $b_{\varphi}$ are measurable [4, Proposition 5.1].

Furthermore, we define the right-continuous inverse at point $t \in \Omega$

$$
\varphi^{-1}(t, u):=\inf \{v \geq 0: \varphi(t, v)>u\} .
$$


Properties of $\varphi^{-1}$ for a Young function $\varphi$ have been collected in [11, Lemma 3.1].

The following basic relation between the norm and the modular will be used frequently through the paper

$$
\|x\|_{\varphi} \leq 1 \Rightarrow I_{\varphi}(x) \leq\|x\|_{\varphi}
$$

for $x \in L^{\varphi}$ (see [15, Theorem 1.1]). More information on Musielak-Orlicz and Orlicz spaces can be found for example in $[7-9,19]$.

\section{Auxiliary results}

Recall that our goal is to describe the space of pointwise multipliers $M\left(L^{\varphi_{1}}, L^{\varphi}\right)$ between two Musielak-Orlicz spaces and thus we will operate on two MusielakOrlicz functions $\varphi, \varphi_{1}$, both defined over the same measure space $\Omega$. The result will be given in terms of the third Musielak-Orlicz function $\varphi \ominus \varphi_{1}$ - the Young conjugate of $\varphi_{1}$ with respect to $\varphi$. In order to define it we need to introduce the following decomposition of the continuous part of the domain $\Omega$ depending on behaviour of both $\varphi, \varphi_{1}$. Let $\varphi, \varphi_{1}$ be two Musielak-Orlicz functions. We define the following sets:

$$
\begin{aligned}
\Omega_{0,0} & :=\left\{t \in \Omega^{c}: b_{\varphi_{1}}(t)=b_{\varphi}(t)=\infty\right\}, \\
\Omega_{0, \infty} & :=\left\{t \in \Omega^{c}: b_{\varphi_{1}}(t)=\infty, b_{\varphi}(t)<\infty\right\}, \\
\Omega_{\infty, 0} & :=\left\{t \in \Omega^{c}: 0<b_{\varphi_{1}}(t)<\infty, b_{\varphi}(t)=\infty\right\}, \\
\Omega_{\infty, \infty} & :=\left\{t \in \Omega^{c}: 0<b_{\varphi_{1}}(t)<\infty, b_{\varphi}(t)<\infty\right\}, \\
\Omega_{\infty} & :=\Omega_{\infty, \infty} \cup \Omega_{\infty, 0} .
\end{aligned}
$$

Given two Musielak-Orlicz functions $\varphi, \varphi_{1}$ over the same measure space $\Omega$, the Young conjugate of $\varphi_{1}$ with respect to $\varphi$ is defined as

$$
\begin{aligned}
\varphi & \ominus \varphi_{1}(t, u) \\
& := \begin{cases}\sup \left\{\varphi(t, s u)-\varphi_{1}(t, s): 0 \leq s<b_{\varphi_{1}}(t)\right\} & \text { if } t \in \Omega^{c}, \\
\sup \left\{\varphi(t, s u)-\varphi_{1}(t, s): 0 \leq s \leq \min \left\{1 / \varphi^{-1}\left(t, \frac{1}{\mu(\{t\})}\right), \frac{b_{\varphi_{1}}(t)}{2}\right\}\right\} & \text { if } t \in \Omega^{a} .\end{cases}
\end{aligned}
$$

Observe firstly that such defined function $\varphi \ominus \varphi_{1}$ satisfies $b_{\varphi \ominus \varphi_{1}}(t)>0$ for each $t \in \Omega^{a}$. Moreover, it is easy to see, that for $t \in \Omega_{0, \infty}$

$$
\varphi \ominus \varphi_{1}(t, u)= \begin{cases}0 & \text { if } u=0 \\ \infty & \text { if } u>0\end{cases}
$$

In consequence,

$$
\operatorname{supp}\left(L^{\varphi \ominus \varphi_{1}}\right)=\left(\Omega_{0,0} \cup \Omega_{\infty} \cup \Omega^{a}\right) \cap \operatorname{supp}\left(L^{\varphi}\right)
$$

It may be instructive to realize what is $\varphi \ominus \varphi_{1}$, when $\varphi, \varphi_{1}$ are Nakano functions. 
Example 1 Let $p, q: \Omega \rightarrow[1, \infty)$ be two measurable functions and define $\varphi(t, u)=$ $\frac{1}{q(t)} u^{q(t)}, \varphi_{1}(t, u)=\frac{1}{p(t)} u^{p(t)}$ for $t \in \Omega, u \geq 0$. Assume that $q(t) \leq p(t)$ for a.e. $t \in \Omega$. One can easily calculate that

$$
\varphi \ominus \varphi_{1}(t, u)=\frac{1}{r(t)} u^{r(t)}
$$

where $\frac{1}{p(t)}+\frac{1}{r(t)}=\frac{1}{q(t)}$ for a.e. $t \in \Omega$.

Finally, we shall see that $\varphi \ominus \varphi_{1}$ satisfies assumptions of Musielak-Orlicz functions.

Lemma 2 Given two Musielak-Orlicz functions $\varphi, \varphi_{1}$ over the same measure space $\Omega$, the function $\varphi \ominus \varphi_{1}$ is also the Musielak-Orlicz function on $\Omega$.

Proof It is already known that $\varphi \ominus \varphi_{1}(t, \cdot)$ is a Young function for a.e. $t \in \Omega$ (see [11, 13]). Thus we need only to prove measurability of $\varphi \ominus \varphi_{1}(\cdot, u)$ for each $u \geq 0$. Each component of $\Omega$ may be considered separately. We will explain only situation on $\Omega_{\infty}$, since the remaining cases are either simpler, or evident. Firstly we observe that for each $u \geq 0$ and $t \in \Omega_{\infty}$

$$
\begin{aligned}
\varphi \ominus \varphi_{1}(t, u) & =\sup \left\{\varphi(t, s u)-\varphi_{1}(t, s): 0 \leq s<b_{\varphi_{1}}(t)\right\} \\
& =\sup \left\{\varphi\left(t, u b_{\varphi_{1}}(t) v\right)-\varphi_{1}\left(t, b_{\varphi_{1}}(t) v\right): 0 \leq v<1\right\} \\
& =\sup \left\{\varphi\left(t, u b_{\varphi_{1}}(t) v\right)-\varphi_{1}\left(t, b_{\varphi_{1}}(t) v\right): v \in \mathbb{Q} \cap[0,1)\right\} .
\end{aligned}
$$

However, functions $\varphi\left(\cdot, u b_{\varphi_{1}}(\cdot) v\right)$ and $\varphi_{1}\left(\cdot, b_{\varphi_{1}}(\cdot) v\right)$ are measurable by properties of Musielak-Orlicz functions. In consequence, $\varphi \ominus \varphi_{1}(\cdot, u)$ is measurable, as the supremum of countable collection of measurable functions.

In the proof of the main theorem, we are going to imitate inductive argument used in [5] and in [13]. In order to do it we need a kind of decomposition of the measure space $\Omega$. The following two lemmas provide it.

Lemma 3 Let $(\Omega, \Sigma, \mu)$ be non-atomic and let $\varphi$ be a Musielak-Orlicz function such that $b_{\varphi}(t)=\infty$ for $\mu$-a.e. $t \in \Omega$. For each a $>0$ there exists a sequence of pairwise disjoint measurable sets $\left(A_{n}\right)$ such that $\bigcup_{n \in \mathbb{N}} A_{n}=\Omega$ and

$$
\left\|\chi_{A_{n}}\right\|_{\varphi} \leq \frac{1}{a}
$$

for every $n \in \mathbb{N}$.

Proof Fix $a>0$. Define the sets

$$
B_{n}:=\{t \in \Omega: n-1 \leq \varphi(t, a)<n\}
$$

for $n \in \mathbb{N}$. Evidently, each $B_{n}$ is measurable, since the function $\varphi(\cdot, a)$ is measurable. Moreover $\bigcup_{n \in \mathbb{N}} B_{n}=\Omega$ and $\left(B_{n}\right)$ is a sequence of pairwise disjoint sets. Since 
we operate on a non-atomic measure space, each $B_{n}$ may be divided further into a sequence (finite or not) of pairwise disjoint sets $\left(C_{j}^{n}\right)_{j \in I_{n}}$ such that $\bigcup_{j \in I_{n}} C_{j}^{n}=B_{n}$ and $\mu\left(C_{j}^{n}\right) \leq \frac{1}{n}$ for each $j \in I_{n}$. In consequence, we have for $n \in \mathbb{N}$ and $j \in I_{n}$

$$
\begin{aligned}
I_{\varphi}\left(a \chi_{C_{j}^{n}}\right) & =\int_{C_{j}^{n}} \varphi(t, a) d \mu(t) \\
& \leq \mu\left(C_{j}^{n}\right) \sup _{t \in C_{j}^{n}} \varphi(t, a) \leq 1 .
\end{aligned}
$$

It follows that

$$
\left\|\chi_{C_{j}^{n}}\right\|_{\varphi} \leq \frac{1}{a}
$$

for every $n \in \mathbb{N}$ and $j \in I_{n}$. Finally, we get the desired sequence $\left(A_{n}\right)$ just by rearranging the (doubly indexed) sequence $\left(C_{j}^{n}\right)$.

Lemma 4 Let $(\Omega, \Sigma, \mu)$ be non-atomic and let $\varphi$ be a Musielak-Orlicz function such that $0<b_{\varphi}(t)<\infty$ for $\mu$-a.e. $t \in \Omega$. There exists a sequence of pairwise disjoint measurable sets $\left(A_{n}\right)$ such that $\bigcup_{n \in \mathbb{N}} A_{n}=\Omega$ and for each $n \in \mathbb{N}$

$$
\left\|\chi_{A_{n}}\right\|_{\varphi} \leq \frac{2}{\operatorname{ess} \sup _{t \in A_{n}}\left\{b_{\varphi}(t)\right\}}
$$

Proof For each $k \in \mathbb{Z}$ define

$$
B_{k}:=\left\{t \in \Omega: 2^{k-1}<b_{\varphi}(t) \leq 2^{k}\right\} .
$$

Evidently, sets $B_{k}$ are measurable, since $b_{\varphi}$ is a measurable function. Next, for each $k \in \mathbb{Z}$ and $n \in \mathbb{N}$ we define

$$
B_{k, n}:=\left\{t \in B_{k}: n-1 \leq \varphi\left(t, 2^{k-1}\right)<n\right\} .
$$

Then the doubly indexed sequence $\left(B_{k, n}\right)$ consists of pairwise disjoint measurable sets such that $\bigcup_{n \in \mathbb{N}, k \in \mathbb{Z}} B_{k, n}=\Omega$. Denote

$$
I:=\left\{(k, n) \in \mathbb{Z}^{2}: B_{k, n} \neq \emptyset\right\}
$$

For each $(k, n) \in I$ we can further decompose $B_{k, n}$ into a (finite or not) sequence $\left(C_{j}^{k, n}\right)_{j \in I_{k, n}}$ of pairwise disjoint measurable sets in such a way that $\bigcup_{j \in I_{k, n}} C_{j}^{k, n}=B_{k, n}$ and $\mu\left(C_{j}^{k, n}\right) \leq \frac{1}{n}$ for each $j \in I_{k, n}$. Finally, for every $(k, n) \in I$ and $j \in I_{k, n}$ we have 


$$
\begin{aligned}
I_{\varphi}\left(2^{k-1} \chi_{C_{j}^{k, n}}\right) & =\int_{C_{j}^{k, n}} \varphi\left(t, 2^{k-1}\right) d \mu(t) \\
& \leq \mu\left(C_{j}^{k, n}\right) \sup _{t \in C_{j}^{k, n}} \varphi\left(t, 2^{k-1}\right) \leq 1 .
\end{aligned}
$$

In consequence,

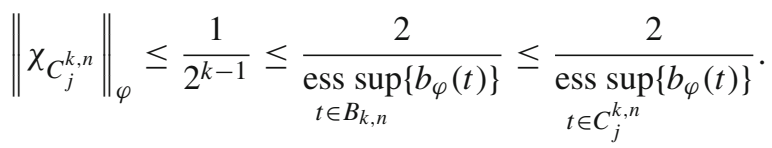

Similarly as before, the desired sequence is obtained after rearranging the (triple indexed) sequence $\left(C_{j}^{k, n}\right)$.

Fact 5 If a Musielak-Orlicz function $\varphi$ is such that $b_{\varphi}(t)<\infty$ for a.e. $t \in \Omega$, then

$$
L^{\varphi} \subset L^{\infty}\left(1 / b_{\varphi}\right)
$$

Proof Let $0 \leq y \notin L^{\infty}\left(1 / b_{\varphi}\right)$. For each $n \in \mathbb{N}$ we define sets

$$
A_{n}=\left\{t \in \Omega: n \leq \frac{y(t)}{b_{\varphi}(t)}\right\}
$$

Then there is $N \in \mathbb{N}$ such that $\mu\left(A_{n}\right)>0$ for $n \geq N$. Fix $a>0$ and choose $n \geq N$ satisfying an $>2$. We can see that

$$
n b_{\varphi} \chi_{A_{n}} \leq y
$$

In consequence,

$$
I_{\varphi}(a y) \geq I_{\varphi}\left(a n b_{\varphi} \chi_{A_{n}}\right) \geq I_{\varphi}\left(2 b_{\varphi} \chi_{A_{n}}\right)=\infty
$$

Since $a>0$ was arbitrary we conclude that $y \notin L^{\varphi}$.

Lemma 6 Let $(\Omega, \Sigma, \mu)$ be non-atomic and let $\varphi, \varphi_{1}$ be two Musielak-Orliczfunctions such that $0<b_{\varphi_{1}}(t)<\infty$ and $0<b_{\varphi}(t)<\infty$ for $\mu$-a.e. $t \in \Omega$. Then

$$
M\left(L^{\varphi_{1}}, L^{\varphi}\right) \subset L^{\infty}\left(b_{\varphi_{1}} / b_{\varphi}\right)
$$

Proof Let $0 \leq y \notin L^{\infty}(v)$, where $v(t):=\frac{b_{\varphi_{1}}(t)}{b_{\varphi}(t)}$. For each $n \in \mathbb{N}$ we define

$$
A_{n}=\{t \in \Omega: n \leq y(t) v(t)<n+1\} .
$$


Then there exist infinitely many $n \in \mathbb{N}$ for which $\mu\left(A_{n}\right)>0$. Denote the set of such $n$ 's by $I$. Next, since $\Omega$ is non-atomic, for each $n \in I$ there is $B_{n} \subset A_{n}$ such that $\mu\left(B_{n}\right)>0$ and

$$
\int_{B_{n}} \varphi_{1}\left(t, \frac{b_{\varphi_{1}}(t)}{2}\right) d \mu(t) \leq \frac{1}{2^{n}}
$$

We define

$$
f(t):=\sum_{n=1}^{\infty} \frac{b_{\varphi_{1}}(t)}{2} \chi_{B_{n}}
$$

Then

$$
I_{\varphi_{1}}(f)=\int \varphi_{1}(t, f(t)) d \mu(t)=\sum_{n=1}^{\infty} \int_{B_{n}} \varphi_{1}\left(t, \frac{b_{\varphi_{1}}(t)}{2}\right) d \mu(t) \leq 1
$$

It means that $f \in L^{\varphi_{1}}$ and $\|f\|_{\varphi_{1}} \leq 1$. However,

$$
y(t) f(t) \geq \frac{1}{2} y(t) b_{\varphi_{1}}(t) \geq \frac{n}{2} b_{\varphi}(t) \quad \text { for a.e. } t \in B_{n},
$$

which implies that $y f \notin L^{\varphi}$, since $L^{\varphi} \subset L^{\infty}\left(\frac{1}{b_{\varphi}}\right)$ by Fact 5. Consequently, $y \notin$ $M\left(L^{\varphi_{1}}, L^{\varphi}\right)$ and the proof is finished.

Lemma 7 Suppose $(\Omega, \Sigma, \mu)$ is non-atomic and let $\varphi, \varphi_{1}$ be Musielak-Orlicz functions such that supp $L^{\varphi_{1}}=\Omega$. Then

$$
\mu\left(\operatorname{supp} M\left(L^{\varphi_{1}}, L^{\varphi}\right) \backslash \Omega_{0,0} \cup \Omega_{\infty}\right)=0 .
$$

Proof We need only to show that $\mu\left(\Omega_{0, \infty} \cap \operatorname{supp} M\left(L^{\varphi_{1}}, L^{\varphi}\right)\right)=0$. Suppose, for a contrary, there exists $A \subset \Omega_{0, \infty}$ such that $\mu(A)>0$ and $\chi_{A} \in M\left(L^{\varphi_{1}}, L^{\varphi}\right)$. Let $C \subset A$ be chosen in such a way that $\mu(C)>0$ and $\inf _{t \in C} b_{\varphi}(t)=\delta>0$. From Lemma 3 it follows that for each $n \in \mathbb{N}$ there exists $A_{n} \subset C$ such that $\mu\left(A_{n}\right)>0$ and

$$
\left\|\chi_{A_{n}}\right\|_{\varphi_{1}} \leq \frac{1}{n}
$$


Moreover, by Fact 5, we know that $L^{\varphi}\left[\Omega_{0, \infty}\right] \subset L^{\infty}\left(\frac{1}{b_{\varphi}}\right)\left[\Omega_{0, \infty}\right]$ with some inclusion constant $c>0$. It means

$$
\begin{aligned}
\left\|\chi_{A_{n}}\right\|_{\varphi} & \geq c^{-1}\left\|\chi_{A_{n}}\right\|_{L^{\infty}\left(\frac{1}{b_{\varphi}}\right)} \\
& \geq c^{-1} \sup _{t \in A_{n}} \frac{1}{b_{\varphi}(t)} \\
& =\frac{1}{c \inf _{t \in A_{n}} b_{\varphi}(t)} \\
& \geq \frac{1}{c \inf _{t \in C} b_{\varphi}(t)}=\frac{1}{c \delta} .
\end{aligned}
$$

Finally, for each $n \in \mathbb{N}$ define $x_{n}:=n \chi_{A_{n}}$. Then $\left\|x_{n}\right\|_{L^{\varphi_{1}}} \leq 1$ and it follows

$$
\left\|\chi_{A}\right\|_{M} \geq\left\|x_{n} \chi_{A}\right\|_{\varphi}=\left\|n \chi_{A_{n}}\right\|_{\varphi} \geq \frac{n}{c \delta}
$$

for each $n \in \mathbb{N}$. In consequence, $\chi_{A} \notin M\left(L^{\varphi_{1}}, L^{\varphi}\right)$ which contradicts our assumption.

Of course, the supremum in definition of function $\varphi \ominus \varphi_{1}$ need not be attained. To avoid such a situation, we introduce a truncated version of $\varphi \ominus \varphi_{1}$ (cf. [13, Definition 1]). Namely, for $a>0$ we define the function $\varphi \ominus_{a} \varphi_{1}$ in the following way

$$
\begin{aligned}
& \varphi \ominus_{a} \varphi_{1}(t, u) \\
& := \begin{cases}\sup \left\{\varphi(t, s u)-\varphi_{1}(t, s): 0 \leq s \leq a\right\} & \text { if } t \in \Omega_{0,0} \cup \Omega_{0, \infty} \\
\sup \left\{\varphi(t, s u)-\varphi_{1}(t, s): 0 \leq s \leq \frac{a}{a+1} b_{\varphi_{1}}(t)\right\} & \text { if } t \in \Omega_{\infty} \\
\sup \left\{\varphi(t, s u)-\varphi_{1}(t, s): 0 \leq s \leq \min \left\{1 / \varphi^{-1}\left(t, \frac{1}{\mu(\{t\})}\right), \frac{b_{\varphi_{1}}(t)}{2}\right\}\right\} & \text { if } t \in \Omega^{a}\end{cases}
\end{aligned}
$$

Using exactly the same reasoning as in the proof of Lemma 2 one can see that $\varphi_{a} \ominus_{1}$ is the Musielak-Orlicz function over $\Omega$. Furthermore, it is easy to see that

$$
b_{\varphi \ominus_{a} \varphi_{1}}(t)=\frac{(a+1) b_{\varphi}(t)}{a b_{\varphi_{1}}(t)}
$$

for $t \in \Omega_{\infty}$.

Lemma 8 Let $(\Omega, \Sigma, \mu)$ be non-atomic and let $\varphi, \varphi_{1}$ be Musielak-Orlicz functions such that supp $L^{\varphi_{1}}=\Omega$. If $A \subset \operatorname{supp}^{\varphi} \backslash \Omega_{\infty, 0}$ is a set of positive measure and numbers $a>1, u>0$ satisfy $\varphi \ominus_{a} \varphi_{1}\left(t, \frac{3}{2} u\right)<\infty$ for a.e. $t \in A$, then the function $x: A \rightarrow \mathbb{R}_{+}$, defined by

$$
x(t):=\max \left\{0 \leq v \leq \min \left\{a, \frac{a}{a+1} b_{\varphi_{1}}(t)\right\}: \varphi_{1}(t, v)+\varphi \ominus_{a} \varphi_{1}(t, u)=\varphi(t, u v)\right\},
$$

is measurable. 
Proof Without loss of generality we may assume that $\varphi_{1}(t, \cdot), \varphi(t, \cdot)$ are Young functions for each $t \in A$. Fix $u>0$ and $a>1$ satisfying

$$
\varphi \ominus_{a} \varphi_{1}\left(t, \frac{3}{2} u\right)<\infty \text { for a.e.t } \in A
$$

and let $x$ be like in the statement. Let $\left(r_{k}\right)$ be a dense sequence in $[0, a]$. For each $k, n \in \mathbb{N}$ define

$$
B_{k}^{n}:=\left\{t \in A: r_{k} \leq \frac{a}{a+1} b_{\varphi_{1}}(t), \varphi_{1}\left(t, r_{k}\right)+\varphi \ominus_{a} \varphi_{1}(t, u)-\varphi\left(t, u r_{k}\right)<1 / n\right\}
$$

and

$$
q_{k}^{n}:=r_{k} \chi_{B_{k}^{n}}
$$

Just notice that by the definition of $\varphi \ominus_{a} \varphi_{1}$

$$
0 \leq \varphi_{1}(t, v)+\varphi \ominus_{a} \varphi_{1}(t, w)-\varphi(t, w v)
$$

for a.e. $t \in \Omega$ and $w, v \geq 0$. Therefore,

$$
\varphi\left(t, u r_{k}\right)<\infty
$$

because for every $k \in \mathbb{N}$ we have $\varphi_{1}\left(t, r_{k}\right)<\infty$ and $\varphi \ominus_{a} \varphi_{1}(t, u)<\infty$. Of course, functions $q_{k}^{n}$ are measurable, since sets $B_{k}^{n}$ are measurable. We will show that

$$
x=\limsup _{k, n \rightarrow \infty} q_{k}^{n} .
$$

Firstly we will explain the inequality $\lim \sup _{k, n \rightarrow \infty} q_{k}^{n} \leq x$. Suppose, for a contradiction, that for some $t_{0} \in A$ and some $\delta>0$ there holds

$$
\limsup _{k, n \rightarrow \infty} q_{k}^{n}\left(t_{0}\right)>x\left(t_{0}\right)+\delta
$$

This implies that there is a (singly-indexed) sequence $\left(q_{k_{i}}^{n_{i}}\right)$ such that $\min \left\{a, \frac{a}{a+1} b_{\varphi_{1}}\right.$ $\left.\left(t_{0}\right)\right\} \geq q_{k_{i}}^{n_{i}}\left(t_{0}\right)>x\left(t_{0}\right)+\delta, n_{i}, k_{i} \rightarrow \infty$ and

$$
\varphi_{1}\left(t_{0}, u_{q_{k_{i}}^{n_{i}}}\left(t_{0}\right)\right)+\varphi \ominus_{a} \varphi_{1}\left(t_{0}, u\right)-\varphi\left(t_{0}, u_{q_{k_{i}}^{n_{i}}}\left(t_{0}\right)\right)<1 / n_{i}
$$

for each $i=1,2,3, \ldots$ On the other hand, there is a subsequence $\left(q_{j}\right):=\left(q_{k_{i_{j}}}^{n_{i_{j}}}\right)$ of $\left(q_{k_{i}}^{n_{i}}\right)$ and $q_{0}>x\left(t_{0}\right)$ such that $q_{j}\left(t_{0}\right) \rightarrow q_{0}$. However, by (3.5) and continuity of respective functions, we get

$$
\varphi_{1}\left(t_{0}, q_{0}\right)+\varphi \ominus_{a} \varphi_{1}\left(t_{0}, u\right)-\varphi\left(t_{0}, u q_{0}\right)=0
$$


which contradicts maximality of $x\left(t_{0}\right)$ and proves inequality $\lim \sup _{k, n \rightarrow \infty} q_{k}^{n} \leq x$.

To see the opposite inequality fix $t \in A$ and denote

$$
C_{n}:=\left\{0 \leq v \leq \min \left\{a, \frac{a}{a+1} b_{\varphi_{1}}(t)\right\}: \varphi_{1}(t, v)+\varphi \ominus_{a} \varphi_{1}(t, u)-\varphi(t, u v)<1 / n\right\} .
$$

We see that sets $C_{n}$ are open and non-empty, since $x(t) \in C_{n}$ for each $n$. Therefore, one can select a sequence $\left(r_{n_{i}}\right)$ such that $r_{n_{i}} \in C_{i}$ and $r_{n_{i}} \rightarrow x(t)$. Then $t \in B_{n_{i}}^{i}$ for each $i=1,2,3, \ldots$ and, consequently,

$$
x(t) \leq \limsup _{k, n \rightarrow \infty} q_{k}^{n}(t),
$$

which finally proves measurability of $x$.

\section{Pointwise multipliers}

Theorem 9 Let $\varphi, \varphi_{1}$ be Musielak-Orlicz functions over a measure space $(\Omega, \Sigma, \mu)$ and assume that supp $L^{\varphi_{1}}=\Omega$. Then

$$
M\left(L^{\varphi_{1}}, L^{\varphi}\right)=L^{\varphi \ominus \varphi_{1}} .
$$

Proof Without loss of generality we can assume that $\operatorname{supp}\left(L^{\varphi}\right)=\Omega$, since

$$
M\left(L^{\varphi_{1}}, L^{\varphi}\right)\left[\Omega \backslash \operatorname{supp}\left(L^{\varphi}\right)\right]=\{0\}=L^{\varphi \ominus \varphi_{1}}\left[\Omega \backslash \operatorname{supp}\left(L^{\varphi}\right)\right],
$$

where the second equality follows from (3.1). The proof of inclusion

$$
L^{\varphi \ominus \varphi_{1}} \subset M\left(L^{\varphi_{1}}, L^{\varphi}\right)
$$

is the same as in the case of Orlicz spaces and we omit it (see for example [13, Lemma 6]).

We only need to prove the remaining inclusion

$$
M\left(L^{\varphi_{1}}, L^{\varphi}\right) \subset L^{\varphi \ominus \varphi_{1}}
$$

Let $0 \leq y \in M\left(L^{\varphi_{1}}, L^{\varphi}\right)$ be a simple function such that $\|y\|_{M} \leq \frac{1}{4 c}$, where $c \geq 1$ is the constant of inclusion

$$
M\left(L^{\varphi_{1}}, L^{\varphi}\right)\left[\Omega_{\infty, \infty}\right] \subset L^{\infty}\left(b_{\varphi_{1}} / b_{\varphi}\right)\left[\Omega_{\infty, \infty}\right]
$$

(cf. Lemma 6). We will show that

$$
I_{\varphi \ominus_{a} \varphi_{1}}(y) \leq 1
$$

for every $a>1$. 
To prove this inequality, for each $a>1$ we will construct a function $x(t)$ on $\Omega$ and a family of pairwise disjoint sets $\left(A_{n}\right)$ satisfying:

(i) $\varphi \ominus_{a} \varphi_{1}(t, y(t))=\varphi(t, x(t) y(t))-\varphi_{1}(t, x(t))$ for a.e $t \in \Omega$,

(ii) $\left\|x y \chi_{A_{n}}\right\|_{\varphi} \leq \frac{1}{2}$ for each $n \in \mathbb{N}$,

(iii) $\operatorname{supp}\left(M\left(L^{\varphi_{1}}, L^{\varphi}\right)\right) \subset \bigcup_{n \in \mathbb{N}} A_{n}$,

(iv) $x \in L^{\varphi_{1}}$ and $\|x\|_{\varphi_{1}} \leq 1$.

Let $a>1$. Since $y$ is a simple function we can write it in the form

$$
y=\sum_{k=0}^{n} b_{k} \chi_{B_{k}}+\sum_{k=0}^{m} d_{k} \chi_{\left\{\omega_{k}\right\}},
$$

where for every $k$ we have $b_{k}, d_{k}>0, B_{k} \subset \Omega_{\infty} \cup \Omega_{0,0}, \mu\left(B_{k}\right)<\infty$ and $\omega_{k}$ 's are atoms. In order to construct the desired function $x$, we will apply Lemma 8 for each $b_{k}$ and $B_{k}$. First of all we need to show that assumptions of Lemma 8 are fulfilled, i.e. for each $0 \leq k \leq n$ we have $\varphi \ominus_{a} \varphi_{1}\left(t, \frac{3}{2} b_{k}\right)<\infty$ for a.e. $t \in B_{k}$. Let $0 \leq k \leq n$. Then for a.e. $t \in B_{k}$ we have

$$
b_{k}=y(t) \leq \frac{b_{\varphi \ominus \varphi_{1}}(t)}{4} \leq \frac{b_{\varphi \ominus_{a} \varphi_{1}}(t)}{2},
$$

since, by Lemma 6,

$$
\left\|y b_{\varphi \ominus \varphi_{1}}^{-1} \chi_{\Omega_{\infty, \infty}}\right\|_{\infty} \leq c\left\|y \chi_{\Omega_{\infty, \infty}}\right\|_{M} \leq \frac{1}{4}
$$

and

$$
b_{\varphi \ominus_{a} \varphi_{1}}(t)=\infty
$$

for a.e. $t \in \Omega_{0,0} \cup \Omega_{\infty, 0}$. Consequently $\varphi \ominus_{a} \varphi_{1}\left(t, \frac{3}{2} b_{k}\right)<\infty$ for a.e. $t \in B_{k}$. Thus using Lemma 8 for the set $B_{k}$ and the number $b_{k}$ we obtain measurable function $x_{k}(t)$ on $B_{k}$ such that

$$
\varphi \ominus_{a} \varphi_{1}(t, y(t))=\varphi\left(t, x_{k}(t) y(t)\right)-\varphi_{1}\left(t, x_{k}(t)\right)
$$

and $0 \leq x_{k}(t) \leq \min \left\{a, \frac{a}{a+1} b_{\varphi_{1}}(t)\right\}$ for a.e. $t \in B_{k}$.

Now we will consider the atomic part. For every $0 \leq k \leq m$ let $c_{k}>0$ satisfy

$$
0 \leq c_{k} \leq \min \left\{1 / \varphi^{-1}\left(\omega_{k}, \frac{1}{\mu\left(\left\{\omega_{k}\right\}\right)}\right), \frac{b_{\varphi_{1}}\left(\omega_{k}\right)}{2}\right\}
$$

and

$$
\varphi \ominus_{a} \varphi_{1}\left(\omega_{k}, y\left(\omega_{k}\right)\right)=\varphi\left(\omega_{k}, c_{k} y\left(\omega_{k}\right)\right)-\varphi_{1}\left(\omega_{k}, c_{k}\right) .
$$

Such numbers exist, since the supremum in definition of $\varphi \ominus_{a} \varphi_{1}$ is taken over a compact set. 
The function satisfying (i) is defined as

$$
x(t):= \begin{cases}x_{k}(t) & \text { if } t \in B_{k}, 0 \leq k \leq n, \\ c_{k} & \text { if } t=\omega_{k}, 0 \leq k \leq m, \\ 0 & \text { if } t \notin \operatorname{supp}(y)\end{cases}
$$

In the next step we will determine sets $\left(A_{n}\right)$ satisfying (ii) and (iii).

We start with $\Omega_{\infty}$. By Lemma 4 there exists a sequence of pairwise disjoint measurable sets $\left(A_{n}^{1}\right)$ such that $\bigcup_{n \in \mathbb{N}} A_{n}^{1}=\Omega_{\infty}$ and

$$
\left\|\chi_{A_{n}^{1}}\right\|_{\varphi_{1}} \leq \frac{2}{\sup _{t \in A_{n}^{1}}\left\{b_{\varphi_{1}}(t)\right\}}
$$

for every $n \in \mathbb{N}$. Since $0 \leq x(t)<b_{\varphi_{1}}(t)$, we have

$$
\left\|x y \chi_{A_{n}^{1}}\right\|_{\varphi} \leq \sup _{t \in A_{n}^{1}}\left\{b_{\varphi_{1}}(t)\right\}\|y\|_{M}\left\|\chi_{A_{n}^{1}}\right\|_{\varphi_{1}} \leq \frac{1}{2},
$$

and therefore sets $\left(A_{n}^{1}\right)$ satisfy (ii).

Secondly, by Lemma 3, there exists a sequence $\left(A_{n}^{2}\right)$ of pairwise disjoint measurable sets such that $\bigcup_{n \in \mathbb{N}} A_{n}^{2}=\Omega_{0,0}$ and

$$
\left\|\chi_{A_{n}^{2}}\right\|_{\varphi_{1}} \leq \frac{1}{a}
$$

Moreover, we have

$$
\left\|x y \chi_{A_{n}^{2}}\right\|_{\varphi} \leq \frac{a}{2}\left\|\chi_{A_{n}^{2}}\right\|_{\varphi_{1}} \leq \frac{1}{2}
$$

because $x(t) \leq a$.

Considering the atomic part, let's observe that for each $\omega \in \Omega^{a}$

$$
\|x y \chi\{\omega\}\|_{\varphi} \leq \frac{1}{2 \varphi^{-1}\left(\omega, \frac{1}{\mu(\{\omega\})}\right)}\|\chi\{\omega\}\|_{\varphi_{1}}=\frac{1}{2},
$$

where the last equality follows by $\left\|\chi_{\{\omega\}}\right\|_{\varphi_{1}}=\varphi^{-1}\left(\omega, \frac{1}{\mu(\{\omega\})}\right)$. Therefore, we can take atoms as desired sets.

Finally, it is enough to renumerate the sequences $\left(A_{n}^{1}\right),\left(A_{n}^{2}\right),(\{\omega\})_{\omega \in \operatorname{Supp}}\left(M\left(L^{\varphi}{ }^{\varphi}, L^{\varphi}\right)\right)^{a}$ into one sequence $\left(A_{n}\right)$. By Lemma 7 ,

$$
\operatorname{supp}\left(M\left(L^{\varphi_{1}}, L^{\varphi}\right)\right) \subset \bigcup_{n \in \mathbb{N}} A_{n},
$$


thus the construction of desired sets $\left(A_{n}\right)$ is finished.

It just left to show that (iv) is fulfilled, i.e.

$$
\|x\|_{\varphi_{1}} \leq 1
$$

In order to prove it, we define functions $x_{n}:=\sum_{k=1}^{n} x \chi_{A_{k}}$ and we will inductively show that

$$
I_{\varphi_{1}}\left(x_{n}\right) \leq \frac{1}{2}
$$

Since $x_{n} \uparrow x$ a.e., from the Fatou property, it will follow that $x \in L^{\varphi_{1}}$ and

$$
\|x\|_{\varphi_{1}} \leq \sup _{n}\left\|x_{n}\right\|_{\varphi_{1}} \leq 1
$$

Firstly we need to show that for every $k \in \mathbb{N}$ there holds

$$
\left\|x \chi_{A_{k}}\right\|_{\varphi_{1}} \leq \frac{1}{2}
$$

From the equality

$$
\varphi \ominus_{a} \varphi_{1}(t, y(t))=\varphi(t, x(t) y(t))-\varphi_{1}(t, x(t))
$$

we obtain two inequalities

$$
\begin{aligned}
\varphi_{1}(t, x(t)) & \leq \varphi(t, x(t) y(t)) \text { for a.e. } t \in \Omega, \\
\varphi \ominus_{a} \varphi_{1}(t, y(t)) & \leq \varphi(t, x(t) y(t)) \text { for a.e. } t \in \Omega .
\end{aligned}
$$

From (4.6) and by inequality $\left\|x y \chi_{A_{k}}\right\|_{\varphi} \leq \frac{1}{2}$ we have

$$
I_{\varphi_{1}}\left(x \chi_{A_{k}}\right)=\int_{A_{k}} \varphi_{1}(t, x(t)) d \mu(t) \leq \int_{A_{k}} \varphi(t, y(t) x(t)) d \mu(t)=I_{\varphi}\left(y x \chi_{A_{k}}\right) \leq \frac{1}{2}
$$

for every $k \in \mathbb{N}$, where the last inequality follows from (2.2).

In particular, $I_{\varphi_{1}}\left(x_{1}\right) \leq \frac{1}{2}$, and we can proceed with the induction. Let $n \geq 1$ and suppose that

$$
I_{\varphi_{1}}\left(x_{n}\right) \leq \frac{1}{2}
$$

We have

$$
I_{\varphi_{1}}\left(x_{n+1}\right)=I_{\varphi_{1}}\left(x_{n}\right)+I_{\varphi_{1}}\left(x \chi_{A_{n+1}}\right) \leq 1
$$

and thus $\left\|x_{n+1}\right\|_{\varphi_{1}} \leq 1$.

Similarly, as in inequality (4.8), we obtain

$$
I_{\varphi_{1}}\left(x_{n+1}\right) \leq I_{\varphi}\left(y x_{n+1}\right) \leq \frac{1}{2},
$$


by $\left\|y x_{n+1}\right\|_{\varphi} \leq \frac{1}{2}\left\|x_{n+1}\right\|_{\varphi_{1}} \leq \frac{1}{2}$. It means that (4.5) is proved and (iv) follows.

Finally, we are ready to show that $I_{\varphi \ominus_{a} \varphi_{1}}(y) \leq 1$. We have

$$
\|y x\|_{\varphi} \leq\|y\|_{M}\|x\|_{\varphi_{1}} \leq \frac{1}{2}
$$

and from inequality (4.7) we obtain

$$
I_{\varphi \ominus_{a} \varphi_{1}}(y)=\int \varphi \ominus_{a} \varphi_{1}(t, y(t)) d \mu(t) \leq \int \varphi(t, y(t) x(t)) d \mu(t)=I_{\varphi}(y x) \leq 1 .
$$

Clearly, $\varphi \ominus_{a} \varphi_{1}(t, y(t)) \uparrow \varphi \ominus \varphi_{1}(t, y(t))$ for a.e. $t \in \Omega$ when $a \uparrow \infty$. Applying the Fatou lemma we have

$$
I_{\varphi \ominus \varphi_{1}}(y)=\int_{\Omega} \varphi \ominus \varphi_{1}(t, y(t)) d \mu(t) \leq \liminf _{a \rightarrow \infty} \int_{\Omega} \varphi \ominus_{a} \varphi_{1}(t, y(t)) d \mu(t) \leq 1,
$$

which proves the inequality (4.1). It means that $y \in L^{\varphi \ominus \varphi_{1}}$ and

$$
\|y\|_{\varphi \ominus \varphi_{1}} \leq 1
$$

Concluding, if $0 \leq y \in M\left(L^{\varphi_{1}}, L^{\varphi}\right)$ is a simple function, then $y \in L^{\varphi \ominus \varphi_{1}}$ and

$$
\|y\|_{\varphi \ominus \varphi_{1}} \leq 4 c\|y\|_{M}
$$

Thus the theorem is proved for positive simple functions. We will once again use the Fatou property to complete the argument for an arbitrary function.

Let now $y \in M\left(L^{\varphi_{1}}, L^{\varphi}\right)$ be arbitrary. There exists a sequence of simple functions $\left(y_{n}\right)$ such that $0 \leq y_{n} \uparrow|y|$ a.e. on $\Omega$. Since $M\left(L^{\varphi_{1}}, L^{\varphi}\right)$ is a Banach ideal space, $\left\|y_{n}\right\|_{M} \leq\|y\|_{M}$ for every $n \in \mathbb{N}$. From the Fatou property of $L^{\varphi \ominus \varphi_{1}}$ we have $y \in$ $L^{\varphi \ominus \varphi_{1}}$ and

$$
\|y\|_{\varphi \ominus \varphi_{1}} \leq \sup _{n \in \mathbb{N}}\left\|y_{n}\right\|_{\varphi \ominus \varphi_{1}} \leq 4 c\left\|y_{n}\right\|_{M} \leq 4 c\|y\|_{M},
$$

which finishes the proof.

In the special case of variable exponent spaces we have the following corollary. It has been recently proved in [10] using elementary methods. Recall that the variable exponent space (or Nakano space) is defined as $L^{p(\cdot)}:=L^{\varphi}$, where $\varphi(t, u)=u^{p(t)}$, for a measurable function $p: \Omega \rightarrow[1, \infty)$.

Corollary 10 Let $(\Omega, \Sigma, \mu)$ be non-atomic and let $p, q: \Omega \rightarrow[1, \infty)$ be two measurable functions satisfying $q(t) \leq p(t)$ for $\mu$-a.e. $t \in \Omega$. Then

$$
M\left(L^{p(\cdot)}, L^{q(\cdot)}\right)=L^{r(\cdot)},
$$

where $\frac{1}{p(t)}+\frac{1}{r(t)}=\frac{1}{q(t)}$ for $\mu$-a.e. $t \in \Omega$. 
Proof First of all, observe that each Nakano space $L^{p(\cdot)}$ may be equivalently defined by the Musielak-Orlicz function $\varphi_{p}(t, u)=\frac{1}{p(t)} u^{p(t)}$. In fact, we see that for $\varphi(t, u)=$ $u^{p(t)}$ there holds

$$
\varphi\left(t, \frac{u}{2}\right)=\left(\frac{u}{2}\right)^{p(t)} \leq \frac{1}{p(t)} u^{p(t)}=\varphi_{p}(t, u) \leq \varphi(t, u)
$$

for each $t \in \Omega$ and $u>0$, which means that $L^{p(\cdot)}=L^{\varphi_{p}}$. Now the proof follows directly from Example 1 and the above theorem.

\section{Pointwise products}

If $\varphi, \varphi_{1}, \varphi_{2}$ are Musielak-Orlicz functions we write $\varphi_{1}^{-1} \varphi_{2}^{-1} \prec \varphi^{-1}$ if there exists a constant $C>0$ such that

$$
C \varphi_{1}^{-1}(t, u) \varphi_{2}^{-1}(t, u) \leq \varphi^{-1}(t, u)
$$

for a.e. $t \in \Omega$ and each $u \geq 0$. Similarly, we write $\varphi_{1}^{-1} \varphi_{2}^{-1} \succ \varphi^{-1}$ if there exists a constant $C>0$ such that for a.e. $t \in \Omega$ and each $u \geq 0$

$$
C \varphi_{1}^{-1}(t, u) \varphi_{2}^{-1}(t, u) \geq \varphi^{-1}(t, u)
$$

Moreover, $\varphi_{1}^{-1} \varphi_{2}^{-1} \approx \varphi^{-1}$ means that $\varphi_{1}^{-1} \varphi_{2}^{-1} \prec \varphi^{-1}$ and $\varphi_{1}^{-1} \varphi_{2}^{-1} \succ \varphi^{-1}$.

Recall the classical Lozanovskii factorization theorem (see [14, Theorem 6], cf. [6]) which says that each Banach ideal space $E$ factorizes $L^{1}$, this is

$$
E \odot M\left(E, L^{1}\right)=L^{1}
$$

Generalizing this idea, for a couple of Banach ideal spaces $E, F$ we say that $E$ factorizes $F$ if

$$
E \odot M(E, F)=F,
$$

(see [12, Section 6] for a discussion of the general factorization problem). Recently the authors proved in [13, Theorem 2] that for a pair of Young functions $\varphi, \varphi_{1}$, the function space $L^{\varphi_{1}}$ may be factorized by $L^{\varphi}$ if and only if

$$
\varphi_{1}^{-1}\left(\varphi \ominus \varphi_{1}\right)^{-1} \approx \varphi^{-1}
$$

That result is based on Theorem 5 in [12], which states that in the case of non-atomic and finite measure space, given three Young functions $\varphi, \varphi_{0}, \varphi_{1}$, there holds

$$
L^{\varphi} \odot L^{\varphi_{1}}=L^{\varphi_{0}}
$$

if and only if

$$
\varphi_{1}^{-1} \varphi_{0}^{-1} \approx \varphi^{-1}
$$


In this section we will show that, in the case of Musielak-Orlicz spaces, the condition (5.1) is sufficient, but not necessary to have the factorization

$$
L^{\varphi} \odot L^{\varphi_{1}}=L^{\varphi_{0}} .
$$

An immediate consequence of Theorem 9 is the following inclusion.

Lemma 11 Let $\varphi, \varphi_{1}$ be Musielak-Orliczfunctions over $(\Omega, \Sigma, \mu)$. If $\operatorname{supp}\left(L^{\varphi_{1}}\right)=\Omega$ then

$$
L^{\varphi} \subset L^{\varphi \ominus \varphi_{1}} \odot L^{\varphi_{1}}
$$

Proof Let $x \in L^{\varphi \ominus \varphi_{1}}$ and $y \in L^{\varphi_{1}}$. Then, since $M\left(L^{\varphi_{1}}, L^{\varphi}\right)=L^{\varphi \ominus \varphi_{1}}$, we see that

$$
x y \in L^{\varphi}
$$

and

$$
\|x y\|_{\varphi} \leq\|x\|_{M}\|y\|_{\varphi_{1}} \leq c\|x\|_{\varphi \ominus \varphi_{1}}\|y\|_{\varphi_{1}} .
$$

Lemma 12 Let $\varphi, \varphi_{0}, \varphi_{1}$ be Musielak-Orlicz functions over $(\Omega, \Sigma, \mu)$. Assume that $\varphi_{1}^{-1} \varphi_{0}^{-1} \succ \varphi^{-1}$ and $\operatorname{supp}^{\varphi_{1}}=\Omega$. Then

$$
L^{\varphi} \subset L^{\varphi_{0}} \odot L^{\varphi_{1}} .
$$

Proof Denote by $c \geq 1$ the constant of inclusion

$$
L^{\varphi}\left[\Omega_{\infty, \infty}\right] \subset L^{\infty}\left(b_{\varphi}^{-1}\right)\left[\Omega_{\infty, \infty}\right]
$$

Let $0 \leq z \in L^{\varphi}$ be such that $\|z\|_{\varphi}=\frac{2}{3 c}$. Put $y(t):=\varphi(t, z(t))$. We have $y(t)<\infty$ a.e., since $z(t) \leq \frac{2}{3} b_{\varphi}(t)$. For $i=0,1$, define

$$
z_{i}(t):= \begin{cases}\varphi_{i}^{-1}(t, y(t)) \sqrt{\frac{z(t)}{\varphi_{0}^{-1}(t, y(t)) \varphi_{1}^{-1}(t, y(t))}} & \text { if } t \in \operatorname{supp}(z) \\ 0 & \text { if } t \notin \operatorname{supp}(z)\end{cases}
$$

Note that $z=z_{0} z_{1}$. We will show that $z_{i} \in L^{\varphi_{i}}$ for $i=0,1$. Let $D>0$ be such that

$$
D \varphi_{1}^{-1}(t, u) \varphi_{0}^{-1}(t, u) \geq \varphi^{-1}(t, u)
$$

We claim that

$$
\varphi_{i}\left(t, \frac{z_{i}(t)}{\sqrt{D}}\right) \leq y(t)
$$

If $y(t)=0$ then

$$
z_{i}(t)=a_{\varphi}(t) \sqrt{\frac{z(t)}{a_{\varphi_{0}}(t) a_{\varphi_{1}}(t)}} \leq a_{\varphi}(t) \sqrt{\frac{a_{\varphi}(t)}{a_{\varphi_{0}}(t) a_{\varphi_{1}}(t)}} \leq a_{\varphi}(t) \sqrt{D}
$$


thus

$$
\varphi_{i}\left(t, \frac{z_{i}(t)}{\sqrt{D}}\right)=0 .
$$

If $y(t)>0$ then

$$
\begin{aligned}
z_{i}(t) & =\varphi_{i}^{-1}(t, y(t)) \sqrt{\frac{z(t)}{\varphi_{0}^{-1}(t, y(t)) \varphi_{1}^{-1}(t, y(t))}} \\
& \leq \varphi_{i}^{-1}(t, y(t)) \sqrt{\frac{D z(t)}{\varphi^{-1}(t, y(t))}} \\
& =\varphi_{i}^{-1}(t, y(t)) \sqrt{\frac{D z(t)}{z(t)}}=\varphi_{i}^{-1}(t, y(t)) \sqrt{D} .
\end{aligned}
$$

Therefore,

$$
\varphi_{i}\left(t, \frac{z_{i}(t)}{\sqrt{D}}\right) \leq \varphi_{i}\left(t, \varphi_{i}^{-1}(t, y(t))\right)=y(t)
$$

and the claim is proved. Integrating both sides in (5.2) we obtain

$$
I_{\varphi_{i}}\left(\frac{z_{i}}{\sqrt{D}}\right) \leq I_{\varphi}(z) \leq 1
$$

for $i=0,1$. It follows, that

$$
\left\|z_{i}\right\|_{\varphi_{i}} \leq \sqrt{D} \leq \sqrt{2 D c\|z\|_{\varphi}}
$$

This means that $z \in L^{\varphi_{0}} \odot L^{\varphi_{1}}$ and

$$
\|z\|_{L^{\varphi_{0}} \odot L^{\varphi_{1}}} \leq 2 D c\|z\|_{\varphi} .
$$

Recall that for Musielak-Orlicz functions $\varphi, \varphi_{1}$, the generalized Young inequality implies that

$$
\varphi_{1}^{-1}\left(\varphi \ominus \varphi_{1}\right)^{-1} \prec \varphi^{-1}
$$

(see for example [11]).

Corollary 13 Let $\varphi, \varphi_{1}$ be Musielak-Orliczfunctions over a measure space $(\Omega, \Sigma, \mu)$. If $\varphi_{1}^{-1}\left(\varphi \ominus \varphi_{1}\right)^{-1} \approx \varphi^{-1}$ then $L^{\varphi_{1}}$ factorizes $L^{\varphi}$.

We finish the paper providing an example, which shows that the opposite implication does not hold. In particular, Theorem 2 in [13] cannot be directly generalized to Musielak-Orlicz spaces. 
Example 14 Consider $\Omega=[0,1 / 2)$ with the Lebesgue measure. Define the following Musielak-Orlicz functions

$$
\begin{aligned}
& \varphi(t, u)=\max \{u-t, 0\}, \\
& \varphi_{1}(t, u)=u,
\end{aligned}
$$

for $t \in \Omega$ and $u \geq 0$. Then $L^{\varphi}=L^{\varphi_{1}}=L^{1}$. Moreover, we have

$$
\varphi \ominus \varphi_{1}(t, u)= \begin{cases}0 & \text { if } 0 \leq u \leq 1 \\ \infty & \text { if } u>1,\end{cases}
$$

thus $L^{\varphi \ominus \varphi_{1}}=L^{\infty}$. In consequence, the factorization

$$
L^{\varphi_{1}} \odot L^{\varphi \ominus \varphi_{1}}=L^{\varphi}
$$

holds. On the other hand an easy computations show that

$$
\left(\varphi \ominus \varphi_{1}\right)^{-1}(t, u)=1, \varphi^{-1}(t, u)=u+t, \varphi_{1}^{-1}(t, u)=u .
$$

We have $\varphi_{1}^{-1}(t, u)\left(\varphi \ominus \varphi_{1}\right)^{-1}(t, u)=u$, thus there is no constant $D$ such that

$$
D \varphi_{1}^{-1}(t, u)\left(\varphi \ominus \varphi_{1}\right)^{-1}(t, u) \geq \varphi^{-1}(t, u)
$$

for every $t \in \Omega$ and $u \geq 0$ (take for example $u=0$ and $t>0$ ). Hence

$$
\varphi_{1}^{-1}\left(\varphi \ominus \varphi_{1}\right)^{-1} \nsucc \varphi^{-1}
$$

Acknowledgements The authors have been partially supported by the Grant $04 / 43 / \mathrm{SBAD} / 0114$ from the Polish Ministry of Science and Higher Education.

Open Access This article is licensed under a Creative Commons Attribution 4.0 International License, which permits use, sharing, adaptation, distribution and reproduction in any medium or format, as long as you give appropriate credit to the original author(s) and the source, provide a link to the Creative Commons licence, and indicate if changes were made. The images or other third party material in this article are included in the article's Creative Commons licence, unless indicated otherwise in a credit line to the material. If material is not included in the article's Creative Commons licence and your intended use is not permitted by statutory regulation or exceeds the permitted use, you will need to obtain permission directly from the copyright holder. To view a copy of this licence, visit http://creativecommons.org/licenses/by/4.0/.

\section{References}

1. Ando, T.: On products of Orlicz spaces. Math. Ann. 140, 174-186 (1960)

2. Bennett, C., Sharpley, R.: Interpolation of Operators. Academic Press, Boston (1988)

3. Calabuig, J.M., Delgado, O., Sánchez Pérez, E.A.: Generalized perfect spaces. Indag. Math. (N.S.) 19(3), 359-378 (2008)

4. Chen, S.: Geometry of Orlicz spaces. Dissertationes Math. (Rozprawy Mat.) 356 (1996), 204. With a preface by Julian Musielak 
5. Djakov, P.B., Ramanujan, M.S.: Multipliers between Orlicz sequence spaces. Turk. J. Math. 24, 313319 (2000)

6. Gillespie, T.A.: Factorization in Banach function spaces. Indag. Math. 43(3), 287-300 (1981)

7. Kamińska, A., Turett, B.: Type and cotype in Musielak-Orlicz spaces. In: Geometry of Banach Spaces, London Math. Soc. Lecture Note Ser. 158, , pp. 165-180. Cambridge Univ. Press, Cambridge (1990)

8. Kamińska, A.: Banach-Saks properties of Musielak-Orlicz and Nakano sequence spaces. Proc. Am. Math. Soc. 142(2), 547-558 (2014)

9. Kamińska, A., Kubiak, D.: The Daugavet property in the Musielak-Orlicz spaces. J. Math. Anal. Appl. 427(2), 873-898 (2015)

10. Karlovich, A.Yu.: The Coburn-Simonenko theorem for Toeplitz operators acting between Hardy type subspaces of different Banach function spaces. Mediterr. J. Math. 15, 91 (2018)

11. Kolwicz, P., Leśnik, K., Maligranda, L.: Pointwise multipliers of Calderón-Lozanovskiĭ spaces. Math. Nachr. 286, 876-907 (2013)

12. Kolwicz, P., Leśnik, K., Maligranda, L.: Pointwise products of some Banach function spaces and factorization. J. Funct. Anal. 266(2), 616-659 (2014)

13. Leśnik, K., Tomaszewski, J.: Pointwise mutipliers of Orlicz function spaces and factorization. Positivity 21(4), 1563-1573 (2017)

14. Lozanovskiı̆, G. Ja.: On some Banach lattices. Sibirsk. Mat. Zh. 10, 584-599 (1969). (Russian); English transl. in Siber. Math. J. 10(3), 419-431 (1969)

15. Maligranda, L.: Orlicz Spaces and Interpolation, Seminars in Mathematics 5. University of Campinas, Campinas (1989)

16. Maligranda, L., Nakai, E.: Pointwise multipliers of Orlicz spaces. Arch. Math. 95(3), 251-256 (2010)

17. Maligranda, L., Persson, L.E.: Generalized duality of some Banach function spaces. Indag. Math. 51(3), 323-338 (1989)

18. Maurey, B.: Théorèmes de factorisation pour les opérateurs linéaires à valeurs dans les espaces $L^{p}$. Astérisque 11, 1-163 (1974)

19. Musielak, J.: Orlicz Spaces and Modular Spaces. Lecture Notes in Mathematics, vol. 1034. Springer, Berlin (1983)

20. Nakai, E.: Pointwise multipliers on Musielak-Orlicz spaces. Nihonkai Math. J. 27(1), 135-146 (2016)

21. Nakai, E.: Pointwise multipliers on Musielak-Orlicz-Morrey spaces. In: Function Spaces and Inequalities, Springer Proc. Math. Stat., 206, pp. 257-281. Springer, Singapore (2017)

22. O'Neil, R.: Fractional integration in Orlicz spaces. I. Trans. Am. Math. Soc. 115, 300-328 (1965)

23. Shragin, I.V.: On certain operators in generalized Orlicz spaces. Dokl. Akad. Nauk SSSR (N.S) 117, 40-43 (1957). (in Russian)

24. Zabreiko, P.P., Rutickii, Ja. B.: Several remarks on monotone functions. Uch. Zap. Kazan. Gos. Univ. 127(1), 114-126 (1967). (in Russian)

Publisher's Note Springer Nature remains neutral with regard to jurisdictional claims in published maps and institutional affiliations. 